\title{
RESEÑA DE LIBROS
}

\author{
D, J. DwYer, People and Housing in the Third World Cities, Londres, \\ Longman, 1979
}

El propósito general del libro es realizar un diagnóstico comparativo de la situación habitacional de la población de las colonias proletarias, callampas, villas miseria, ranchos, asentamientos espontáneos, etc., de algunas ciudades importantes de los países del tercer mundo.

El trabajo presenta implícitamente tres partes. Los tres primeros capitulos se pueden considerar como el contexto general en el cual se enmarca el problema de las colonias proletarias, para usar la expresión con la que comúnmente se coṇoce al fenómeno de la marginalidad espacial en México. El cuarto y quinto capítulo constituyen estudios de casos en los que se realiza un análisis más o menos amplio de la situación habitacional en las colonias proletarias de las ciudades de Caracas, Venezuela y Hong Kong. El sexto capítulo analiza las perspectivas de la "auto-construcción" como la política más adecuada y realista para enfrentar el problema y, por último, en el séptimo capítulo hace una consideración en perspectiva de los "asentamientos espontáneos" o colonias proletarias.

En el capítulo 1 titulado "Una forma urbana principal", el autor ubica el surgimiento de los "asentamientos espontáneos" dentro de las tendencias generales del desarrollo urbano en el mundo. Son interesantes las semejanzas y diferencias que presenta el proceso de urbanización en los países Asiáticos, Africanos y Latinoamericanos que constituyen fundamentalmente el llamado "tercer mundo". Al respecto D. J. Dwyer concluye que no obstante la magnitud actual que presenta el problema de los "asentamientos espontáneos" es necesario destacar dos características del fenómeno:

"Primero, las tasas de crecimiento de los asentamientos espontáneos están todavía aumentando en relación a las tasas de crecimiento de la población urbana total. En otras partes, el problema, tan grande como es, todavía no es tan serio como indudablemente pronto llegará a ser. Segundo, aunque la mayoría de las estimaciones dadas anteriormente son para ciudades muy grandes, los 'asentamientos espontáneos' han llegado a ser la forma urbana principal en los pueblos y ciudades de todos los tamaños en el tercer mundo".

En el capítulo 2 ("Características socioeconómicas") analiza quiénes son los habitantes de los "asentamientos espontáneos". Para este propósito, al 
igual que en todo el libro el autor introduce al lado de las frías estadísticas al respecto, una serie de relatos sobre los habitantes de este tipo de "formas urbanas" que le imprimen al estudio la exacta dimensión humana que el fenómeno objetivamente posee. Adicionalmente, se logra que el libro presente un estilo ameno que lo hace asequible para personas poco especializadas en el tema. El análisis de las características socioeconómicas de los grupos marginales se centra en el estudio de la migración. A este respecto concluye: $i$ ) Es extremadamente inseguro generalizar sobre las características socioeconómicas de los asentamientos espontáneos; ii) De ninguna forma puede suponerse siempre que estos están compuestos por migrantes rurales. Existen importantes movimientos intra-urbanos desde las áreas saturadas dentro de la ciudad; iii) Las características del migrante - edad, sexo, estado civil, etc.- y de la migración —circular, por etapas, etc.- tienen importantes implicaciones para las políticas habitacionales.

El capítulo 3, "Confusión, apatía y esquemas simbólicos", presenta lo inadecuado de las políticas que se habilitan en los países del tercer mundo para hacer frente al problema. Con base en casos concretos de diferentes países, el autor demuestra cómo los programas habitacionales dirigidos a tratar de resolver los problemas de las clases desposeídas presentan fuertes confusiones respecto a la naturaleza del problema que enfrentan. Existe en adición "negligencia" en las políticas oficiales al respecto y, en general, están sustentadas en una serie de "ideales" que "...lejos de ser constructivos reflejan una ciega renuencia a encarar la realidad de la situación habitacional en los países en desarrollo" (p. 102).

En los capítulos 4 ("Políticas de multifamiliares: Caracas") y 5 ("Politicas de multifamiliares: Hong Kong"), se analizan con amplitud las políticas habitacionales en estas dos ciudades que se caracterizan por un fuerte énfasis en la construcción de edificios multifamiliares. Se detectan en estos programas los problemas comúnmente conocidos: explosivos problemas sociales derivados del violento traspaso de las familias que habitan los asentamientos espontáneos a edificios con grandes densidades de población; imposibilidad de que las familias puedan contar con los recursos necesarios para pagar las rentas relativamente elevadas de los departamentos; destrucción de las áreas comúnes; inseguridad de los habitantes; irresolubles problemas de administración; etc. De nuevo el autor constata como la causa de estos problemas la "carencia de planeación cuidadosa", así como una "carencia de principios claros tanto en los estándares físicos como en los sociales" (p. 130).

Para el caso de Venezuela, el autor señala que la búsqueda de una política de vivienda realista da alguna esperanza a su futuro urbano y puede ser también de significación para todo el tercer mundo: "Alguna esperanza indudablemente existe, por ejemplo, en las posibilidades de mejorar los ranchos in situ" (p. 136). Para esto se realizan "planes-pilotos" de vivienda auto-construida de bajo costo con asistencia técnica y préstamos para materiales. A este respecto será necesario hacer notar que en los países donde se han implementado este tipo de planes que constituyen la única alternativa "realista" para tratar de solucionar un problema que se encuentra enraizado en la naturaleza misma de la estructura económica de los países capitalistas del tercer mundo, han tenido básicamente un efecto "sim- 
bólico" y están lejos de resolver el problema habitacional que en el contexto en que están inscritos tienen la característica de ser insoluble.

En el capítulo 6, "Posibilidades de la auto-construcción de vivienda", D. J. Dwyer resume y evalúa los nuevos enfoques de políticas para los problemas habitacionales del tercer mundo haciendo énfasis en los casos de Lima, Perú y Calcuta, India. Analiza el enfoque de J. F. C. Turner cuya conclusión general es que "Los asentamientos espontáneos representan una solución más que un problema: representan la solución para el problema habitacional de los grupos de bajos ingresos de las ciudades de los países subdesarrollados tal como ellos mismos lo conceptualizan" (p. 198). A este enfoque de tinte fuertemente reaccionario le hace algunas críticas aunque, "su considerable mérito permanece" (p. 223).

No obstante señalar la alta prioridad que tiene para la investigación urbana la evaluación de "las fuerzas económicas y sociales que se ponen en juego para conformar los patrones residenciales urbanos en el tercer mundo" (p. 224), en ningún momento se formula la necesidad de modificarlas para estar en posibilidades de solucionar el problema, sino que se inclina para soluciones más o menos convencionales que surgen de su interesante análisis comparativo entre países del capitalismo dependiente (poner énfasis en los conjuntos multifamiliares, garantizar la propiedad legal de la tierra en los asentamientos espontáneos, centrarse en la prestación de servicios de infraestructura y en la auto-construcción, etc.).

Por último, el capítulo 7, "Perspectiva de los asentamientos espontáneos" señala que dadas las tasas de crecimiento de población que presentan la mayor parte de los países del tercer mundo, se experimentarán movimientos masivos de población hacia las ciudades. Las grandes ciudades que "han probado ser los más poderosos magnetos" estarán aún más sobrepobladas. En esta situación "La vida en asentamientos espontáneos llegará a ser la norma para la mayoría de la población urbana" (p. 230).

No hay un concenso claro de cómo evitar o paliar esa perspectiva. Existen dificultades técnicas y políticas para estar en posibilidades de hacerlo, pero el verdadero problema son los bajos ingresos de la población de los países subdesarrollados. Ante esto, no parece probable que los servicios urbanos de corte occidental lleguen "Alguna vez a alcanzar las masas de población de los países del tercer mundo que viven en los asentamientos espontáneos" (p. 236). D. J. Dwyer concluye que se deben desarrollar sistemas de servicios públicos más rudimentarios, lo cual "Es una alternativa triste... pero quizás, actualmente al menos, es la única práctica posible" (p. 236).

Ante las deplorables perspectivas de los asentamientos espontáneos en los países subdesarrollados, el autor se pregunta: " ¿Es esto como debe ser?" El mismo responde: "En una época en que el hombre ha alcanzado la luna, seguramente no".

No obstante, las recomendaciones de política habitacional que esboza el autor está muy lejos de considerar los cambios revolucionarios indispensables para resolver el problema de la marginación social y espacial de los grandes sectores de población de los países del tercer mundo.

Gustavo Garza

El Colegio de México 
MarJory Skowronsky, Abortion and Alternatives, California, Les Femes Xillbras. $1977,145 \mathrm{pp}$.

Existe ya una literatura muy amplia acerca del problema del aborto. Por desgracia, la mayoría de las publicaciones aparecen en idiomas extranjeros. $^{1} \mathrm{El}$ libro que se comenta es un ejemplo de lo anterior.

Todos los días aparecen en los diarios noticias acerca del aborto. Polémicas, conferencias, mesas redondas y seminarios llevados a cabo por profesionales de la política, la ética, la medicina y las leyes. ${ }^{2}$ Vemos pues que el aborto preocupa a todos.

Se dice que es el tema "tabú" de los médicos, puesto que, en México, legalmente no lo pueden ejercer, aunque de hecho les inquieta profundamente como lo muestra la Mesa Redonda sobre el aborto que se llevó a cabo en febrero de 1957 en el Hospital General de la Secretaría de Salubridad y Asistencia en las II Jornadas Médicas.

Los gobiernos adoptan perspectivas distintas de acuerdo a la forma en que afecta esta práctica a sus problemas demográficos y políticos. La liberalizan cuando hay un exceso de población, como en la India; o toman medidas para salvaguardar la vida de las mujeres, cuando éstas no están protegidas por una legislación adecuada, como en Estados Unidos y la Unión Soviética. O, por último, la prescriben cuando la opinión pública es contraria por no haber tomado conciencia de la gravedad del problema, o por la influencia de la Iglesia Católica, como es el caso de México.

Sea que el problema se suscite para evitar el daño a la salud materna o por las consecuencias que tiene para la demografía y la política, el aborto voluntario no es un tema que pueda seguir soslayándose en nuestro país.

La literatura existente agrupa la temática en torno al aborto, jerarquizándola de la siguiente manera:

a) La justificación moral del aborto;

b) La protección legal de la mujer que aborta;

c) El sujeto que decide sobre la práctica concreta.

El libro que se comenta incluye los tres temas anteriores bajo la perspectiva concreta de: la mujer que encara una preñez no deseada. Analiza las posibles alternativas que se le presenta, de allí el título del libro: $E l$ aborto y sus alternativas.

Las alternativas son: dar a luz y cuidar al niño; tenerlo y posteriormente darlo para su adopción, o practicar un aborto.

$\mathrm{La}$ autora investiga cada una de las alternativas presentadas, bajo los puntos de vista legal, médico, psicológico, sociológico y moral.

1 H. L. A. Hart, "Abortion Law Reform: The English Experience; Erody, B. A., "Abortion and the Sanctity of Human Life", ambos en American Philosophical Quarterly, Vol. 10; Además, Wertheimer, R., "Understanding the Abortion Argument"; Judith Jarvis, "A Defence of Abortion"; John Finnis, "The Rights and Wrong of Abortion", estos últimos artículos aparecen en Philosophy \& Public Affairs. Princeton, University Press. New Jersey, (Hay muchísimos más). En español: Revista FEM, Ed. México, 1977, Núm. 2 dedicada al problema del aborto.

2 La reunión interdisciplinaria convocada por el Consejo Nacional de Población, duró varios meses y hay una sesión plenaria en agosto de 1976 para rendir un informe al gobierno. III Jornadas médicas del Hospital General de México, Secretaría de Salubridad y Asistencia, Sesión plenaria sobre el aborto, febrero de 1978. 
Este tipo de estudio es valioso, no sólo para la mujer que sufre la preñez no deseada, también lo es para la que ya tuvo un aborto; para la que no se atrevió a tenerlo; para el hombre o la familia que ayudó a la decisión; para el profesional que se ve o que se ha visto involucrado en el acto del aborto. Esto porque cada una de estas posturas forman el contexto del libro.

Respecto a la primera cuestión: continuar con el embarazo y cuidar al niño, se exploran las consecuencias psicológicas y sociológicas negativas para la mujer, el hijo y la familia.

En el segundo caso se dan indicaciones precisas sobre instituciones norteamericanas que reciben niños en adopción, analizándose las consecuencias de culpabilización de la madre y los problemas que de manera eventual se crean para el hijo y la sociedad.

Para la solución y resolución del aborto, se investigan las legislaciones de una multitud de países que pueden orientar una nueva legislación en nuestro país. Las justificaciones morales de distintas posiciones ideológicas también se revisan. Por último, se analizan los métodos abortivos que constituyen la última palabra en la tecnología de esa materia.

Como tema adicional, se comentan los métodos contraceptivos más modernos y se indican los niveles de efectividad y efectos secundarios posibles.

La autora no da ni respuestas ni recomendaciones para optar por alguno de los caminos planteados. Sin embargo, perfila una tesis básica: las mujeres tienen el derecho, como personas que son, de disponer libremente y sin coacción, de su propio cuerpo y de su afectividad de acuerdo con una previsión inteligente e informada de las consecuencias de su acción.

En ocasiones el libro cae en lo romántico y lo melodramático, pero esto queda compensado por la amplia, precisa y verídica información que contiene en términos de: datos, bibliografía e incluso instituciones que practican abortos y el costo aproximado de la intervención. Esto último, por desgracia, limitado a los Estados Unidos de Norteamérica.

Graciela Hierro 